\title{
Effect of PIP3 on Adhesion Molecules and Adhesion of THP-1 Monocytes to HUVEC Treated with High Glucose
}

\author{
Prasenjit Manna Sushil K. Jain \\ Department of Pediatrics, Louisiana State University Health Sciences Center, Shreveport, USA
}

\section{Key Words}

PIP3 $•$ High glucose $\cdot$ ICAM-1 $\cdot$ CD11a $\bullet$ Monocyte-endothelial cell adhesion

\begin{abstract}
Background: Phosphatidylinositol-3,4,5-triphosphate (PIP3), a well-known lipid second messenger, plays a key role in insulin signaling and glucose homeostasis. Using human umbilical vein endothelial cells (HUVEC) and THP-1 monocytes, we tested the hypothesis that PIP3 can downregulate adhesion molecules and monocyte adhesion to endothelial cells. Methods: HUVEC and monocytes were exposed to high glucose (HG, $25 \mathrm{mM}, 20 \mathrm{~h}$ ) with or without PIP3 (0-20 nM), or PIT-1 (25 $\mu \mathrm{M})$, an inhibitor of PIP3. Results: Both HG and PIT-1 caused a decrease in cellular PIP3 in monocytes and HUVEC compared to controls. Treatment with PIT-1 and HG also increased the ICAM-1 (intercellular adhesion molecule 1) total protein expression as well as its surface expression in HUVEC, CD11a (a subunit of lymphocyte function-associated antigen 1, LFA-1) total protein expression as well as its surface expression in monocytes, and adhesion of monocytes to HUVEC. Exogenous PIP3 supplementation restored the intracellular PIP3 concentrations, downregulated the expression of adhesion molecules, and reduced the adhesion of monocytes to HUVEC treated with HG. Conclusion: This study reports that a decrease in cellular PIP3 is associated with increased expression of adhesion molecules and monocyte-endothelial cell adhesion, and may play a role in the endothelial dysfunction associated with diabetes.
\end{abstract}

Copyright $@ 2014$ S. Karger AG, Basel

\section{Introduction}

The pathogenesis of type 2 diabetes (T2D) is associated with both insulin resistance and impaired glucose metabolism [1]. Endothelial dysfunction has received increasing 
attention as a potential contributor to the pathogenesis of vascular complications in T2D [2]. Hyperglycemia is one of the major causal factors in the development of endothelial dysfunction in T2D [2]. Increasing evidence suggests that the progression of insulin resistance to T2D parallels that of endothelial cell dysfunction to atherosclerosis [2-4]. The importance of insulin's actions on endothelial function were clearly established recently by the findings that mice with a double knockout of apolipoprotein E (apoE) and insulin receptor (IR) on their endothelial cells developed atherosclerosis at 2-3 times greater severity than apoE knockout mice [5].

Membrane bound phosphoinositides (PI) have been implicated in the regulation of a variety of cell signaling processes [6]. Among them, phosphatidylinositol-3,4,5-triphosphate (PIP3) plays a key role in the regulation of glucose transport [7] and glucose metabolism [8]. Deregulation of PI metabolism has been found to cause the onset and progression of several cardiovascular pathologies, ranging from atherosclerosis to heart failure [9]. The presence of insulin receptors on endothelial cells has been reported in the literature [3]. It is reported that hyperglycemia induced uncoupling of downstream insulin signaling at PI3K/AKT in endothelial cells may be responsible for the vascular complications of diabetes [10]. High glucose concentrations have been reported to increase endothelial cell apoptosis [11], permeability [12,13], and monocyte-endothelial cell (EC) adhesion [13, 14]. PI3K/AKT signaling in endothelial cells regulates cell survival, proliferation, microvascular permeability, and angiogenesis [15-17]. Varma et al. [10] reported that high glucose-induced inhibition of the PI3K/AKT pathway causes endothelial cell proliferative dysfunction; the results have also been investigated in the presence of pharmacological inhibitors of PI3K, namely, wortmannin and LY294002. PIP3 has an important function in the regulation of PI3K/AKT signaling. However, at present there is no report in the literature concerning whether PIP3 has a direct effect on endothelial dysfunction and vascular inflammation at the cellular level. Using human umbilical vein endothelial cells (HUVEC) and THP-1 monocytes, this study demonstrates that treatment with high glucose (HG) caused a decrease in intracellular PIP3 levels and increased the expression of adhesion molecules as well as adhesion of monocytes to endothelial cells (EC). Similarly, treatment with a PIP3 inhibitor (PIT-1) also increased the expression of adhesion molecules and monocyte-EC adhesion. Exogenous PIP3 supplementation, however, restored the loss in intracellular PIP3 and downregulated the expression of adhesion molecules as well as monocyte-EC adhesion in HG-treated cells. This suggests a causal role of PIP3 in the regulation of adhesion molecules and the adhesion of monocytes to HUVEC treated with HG.

\section{Material and Methods}

\section{Materials}

CD11a and FITC conjugated CD11a primary antibodies were purchased from Abcam, Inc. (Cambridge, MA). ICAM-1 and FITC conjugated ICAM-1 primary antibodies were purchased from Santa Cruz Biotechnology, Inc. (Santa Cruz, CA). All other chemicals were purchased from Sigma Chemical Co. (St. Louis, MO) unless otherwise mentioned.

\section{Human monocytic cells}

Human THP-1 monocytes were purchased from ATCC. These cells were cultured in RPMI-1640 medium supplemented with $10 \%(\mathrm{v} / \mathrm{v}$ ) heat-inactivated fetal bovine serum, $100 \mathrm{U} / \mathrm{mL}$ penicillin, $100 \mu \mathrm{g} /$ $\mathrm{mL}$ streptomycin, and $2 \mathrm{mM} \mathrm{L}$-glutamine. The culture was maintained at $37^{\circ} \mathrm{C}$ in a humidified atmosphere containing $5 \% \mathrm{CO}_{2}$.

\section{Human umbilical vein endothelial cells (HUVEC)}

Cells were purchased from Lonza Walkersville, Inc. HUVEC were cultured to confluence in EGM-2 media and $5 \% \mathrm{CO}_{2}$ in a $37^{\circ} \mathrm{C}$ humidified atmosphere. The culture was passaged according to standard procedures. For experiments, HUVEC were used within 24 hours after reaching confluence, between passages 3 and 10. The vendor certified that the media had 100 endotoxin concentrations $<0.005 \mathrm{EU} / \mathrm{mL}$. 
Treatment of HUVEC and monocytes with high glucose (HG), PIP3, and PIT-1

In this study, cells were treated with a glucose concentration of $7 \mathrm{mM}$ (control glucose) or $25 \mathrm{mM}$ (high glucose) with and without PIP3. In the human body glucose is continuously degraded and re-formed to maintain a $5 \mathrm{mM}$ blood glucose level. However, in cell culture studies, we observed that incubating cells in medium with a $5 \mathrm{mM}$ glucose concentration for $24 \mathrm{~h}$ caused a decrease in the concentration of glucose in the medium lower than $2 \mathrm{mM}$. In cell culture studies, glucose is metabolized but not replaced. Our experience has shown us that a glucose level of $7 \mathrm{mM}$ does not lead to glucose deficiency after $24 \mathrm{~h}$ of treatment and thus we incubated the control cells in media with a glucose concentration of $7 \mathrm{mM}$. In high glucose studies, cells were exposed to media having a glucose concentration of $25 \mathrm{mM}$ for $20 \mathrm{~h}$. Many previous studies have reported that glucose concentrations as high as $50 \mathrm{mM}$ have been found in the blood of patients with uncontrolled diabetes [18]. It is true that blood glucose levels in patients are not likely to stay as high as $25 \mathrm{mM}$ for $24 \mathrm{~h}$. However, tissue damage in diabetic patients occurs over many years of countless hyperglycemic episodes. Thus, the glucose concentration of $25 \mathrm{mM}$ used in this cell culture study does not seem unreasonable. Anionic phosphatidylinositol phosphate derivatives were delivered across the cell membrane by complexing them with a positive lysine-rich histone carrier following the procedure described by the manufacturer (Echelon Biosciences, Inc., Salt Lake City, UT). Unlabeled Histone H1 (Echelon Biosciences) and PIP3 (Echelon Biosciences) were mixed at a molar ratio of 1:1, vortexed, subjected to sonication, and incubated for 10 minutes at room temperature. The mixture was then added to the medium to a final concentration of 5, 10, or $20 \mathrm{nM}$ for $4 \mathrm{~h}$ followed by HG treatment for the next $20 \mathrm{~h}$. Cells were also treated with PIT-1 (25 $\mathrm{MM}$ ) (Cayman, Ann Arbor, MI), an inhibitor of PIP3, for $20 \mathrm{~h}$. Mannitol was used as an osmolarity control. In the mannitol treated group, cells were exposed to $18 \mathrm{mM}$ mannitol, since the media already contains $7 \mathrm{mM}$ glucose. Cell viability was determined using the Alamar Blue reduction bioassay (Alamar Biosciences, Sacramento, CA). This method is based upon Alamar Blue dye reduction by live cells. After treatment, cells were lysed in RIPA supplemented with protease and phosphatase inhibitors. Lysates were cleared by centrifugation and total protein concentrations were determined using a BCA assay kit (Pierce/Thermo Scientific, Rockford, IL).

\section{Measurement of intracellular PIP3 levels}

Cellular PIP3 concentrations were measured using a PIP3 Mass ELISA Kit (Echelon Biosciences, Inc.; Salt Lake City, UT). Appropriate controls and standards (specified by each manufacturer's kit) were used every time.

\section{Immunoblotting}

All samples contained approximately the same amount of protein $(\sim 10-20 \mu \mathrm{g})$ and were run as $10 \%$ SDS-PAGE and transferred to a nitrocellulose membrane. Membranes were blocked at room temperature for $2 \mathrm{~h}$ in blocking buffer containing 1\% BSA to prevent non-specific binding and then incubated with CD11a (1:20000) or anti-ICAM-1 (1:1000) primary antibodies at $4^{\circ} \mathrm{C}$ overnight. The membranes were washed in TBS-T (50 mmol/L Tris-HCl, pH 7.6, $150 \mathrm{mmol} / \mathrm{L} \mathrm{NaCl}, 0.1 \%$ Tween 20) for $30 \mathrm{~min}$ and incubated with the appropriate HRP conjugated secondary antibody (1:5000 dilution) for $2 \mathrm{~h}$ at room temperature and developed using the ultrasensitive ECL substrate (Millipore, MA). The intensity of each immunoblotting band was measured using the histogram tool of Adobe Photoshop CS5.

\section{Surface analysis of cell membrane proteins by flow cytometry}

Surface analyses of cell membrane proteins (CD11a and ICAM-1) were done using direct staining procedures and flow cytometry as described earlier [19]. Ice cold reagents/solutions and the presence of sodium azide was used to prevent the modulation and internalization of surface receptors. All incubations were all done at $4^{\circ} \mathrm{C}$ in the dark to prevent loss of signal. After treatment, cells were washed in FACS buffer (PBS without $\mathrm{Mg}^{2+}$ and $\mathrm{Ca}^{2+}$, with the addition of $10 \%$ fetal bovine serum and $0.1 \%$ sodium azide), centrifuged, suspended in FACS buffer, and incubated for $2 \mathrm{~h}$ at $4{ }^{\circ} \mathrm{C}$ with FITC conjugated anti-CD11a (Abcam) or anti-ICAM-1 (Santa Cruz Biotechnology) primary antibodies at a 1:50 dilution. After incubation, cells were washed in washing buffer for FACS (PBS containing 1\% BSA and 0.1\% sodium azide) and then suspended in $0.5 \mathrm{~mL}$ FACS buffer for analysis. In each sample, a minimum of 15,000 cells was analyzed (per treatment condition) by using a FACS Calibur flow cytometer (Becton-Dickinson, San Diego, CA) equipped with multicolor analysis capability. Gates were set to exclude nonviable cells, cell debris, and cells of 
Fig. 1. Intracellular PIP3 levels in THP-1 monocytes and HUVEC. A: PIP3 levels in THP-1 monocytes and B: PIP3 levels in HUVEC. Cells were pretreated with PIP3 $(5,10$, or $20 \mathrm{nM})$ for $4 \mathrm{~h}$ followed by HG $(25$ $\mathrm{mM}$ ) exposure for the next $20 \mathrm{~h}$. Cells were also treated with the PIP3 inhibitor PIT-1 $(25 \mu \mathrm{M})$ for $20 \mathrm{~h}$. Values are expressed as mean $\pm \mathrm{SE}(\mathrm{n}=3)$. The difference between * vs \# is considered significant at the $p<0.05$ level.

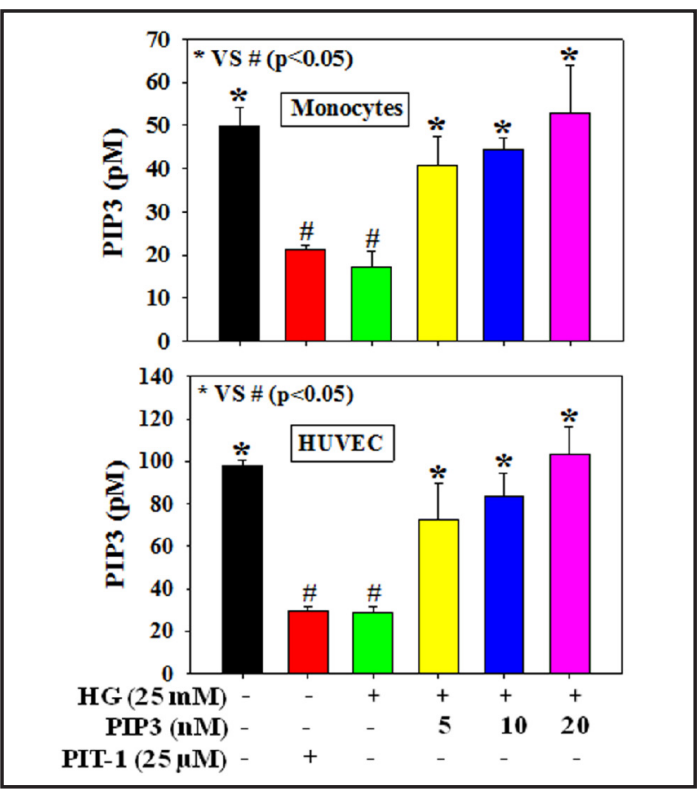

abnormal size and shape. Controls for matched isotypes of primary antibodies were also analyzed to ensure no non-specific binding. Results were expressed as mean fluorescence intensity (MFI) per 15,000 cells.

\section{Monocyte-EC adhesion}

HUVEC were plated and allowed to grow to confluent monolayers. The EC were treated with normal glucose $(7 \mathrm{mM})$ or HG (25 mM, $20 \mathrm{~h})$ either alone or with PIP3 (5, 10, or $20 \mathrm{nM}, 4 \mathrm{~h})$. In another group, normal cells were also treated with PIT-1 (25 $\mu \mathrm{M}, 20 \mathrm{~h})$. Monocytes were loaded with $8 \mu \mathrm{M}$ CellTracker Green (CMFDA) (Invitrogen, Eugene, OR, USA) then treated with HG, PIP3 or PIT-1 at concentrations matching those of EC. After $24 \mathrm{~h}, 1 \times 10^{6}$ monocytic cells were added to the endothelial monolayers and incubated at $37^{\circ} \mathrm{C}$ for $30 \mathrm{~min}$. The non-adherent cells were washed away with endothelial cell media, and adherent cells were lysed in $0.2 \%$ Triton-X for quantification. The fluorescent intensity of the monocytes added to the monolayer was measured at excitation $485 \mathrm{~nm}$ and emission $528 \mathrm{~nm}$. Results were expressed as relative fluorescence intensity (RFI).

\section{Statistical analysis}

Data from cell culture studies were analyzed statistically using one way analysis of variance (ANOVA) with Sigma Stat statistical software (Jandel Scientific, San Rafael, CA). When data passed a normality test, all groups were compared using the Student-Newman-Keuls method. A difference was considered significant at the $p<0.05$ level.

\section{Results}

Figure 1 shows that treatment with HG or the PIP3 inhibitor PIT-1 caused a decrease in intracellular PIP3 concentration in both HUVEC and monocytes compared to those seen in controls. Exogenous PIP3 supplementation (5, 10, or $20 \mathrm{nM}$ ), however, dose-dependently restored the loss in PIP3 in cells treated with HG. Results reported in our earlier study did not show any effect of mannitol supplementation on the PIP3 levels and cell viability compared to controls [20]. Similarly, in the present study we did not observe any effect of mannitol on PIP3 levels and cell viability compared to those of controls (data not shown). Different treatments did not cause any change in cell viability (data not shown).

Figures 2-3 demonstrate the effect of PIP3 on the expression of adhesion molecules, ICAM-1, and CD11a (a sub unit of LFA-1 that plays a central role in leukocyte intercellular adhesion through interactions with its ligand, ICAM, in endothelial cells) in HG-treated endothelial cells and monocytes, respectively. Results demonstrate that HG treatment caused 


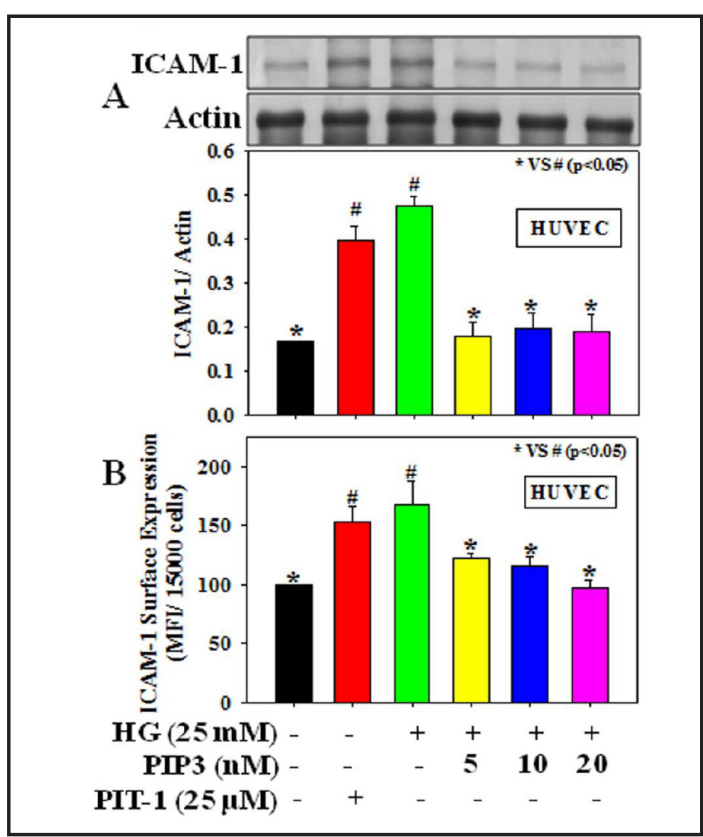

Fig. 2. Effect of PIP3 on ICAM-1 expression in HUVEC exposed to HG. A: ICAM-1 total protein expression and B: ICAM-1 surface expression. Cells were pretreated with PIP3 $(5,10$, or $20 \mathrm{nM})$ for $4 \mathrm{~h}$ followed by HG (25 mM) exposure for the next $20 \mathrm{~h}$. Cells were also treated with the PIP3 inhibitor PIT-1 $(25 \mu \mathrm{M})$ for $20 \mathrm{~h}$. Values are expressed as mean $\pm \mathrm{SE}$ $(\mathrm{n}=3)$. The difference between * vs \# is considered significant at the $p<0.05$ level.

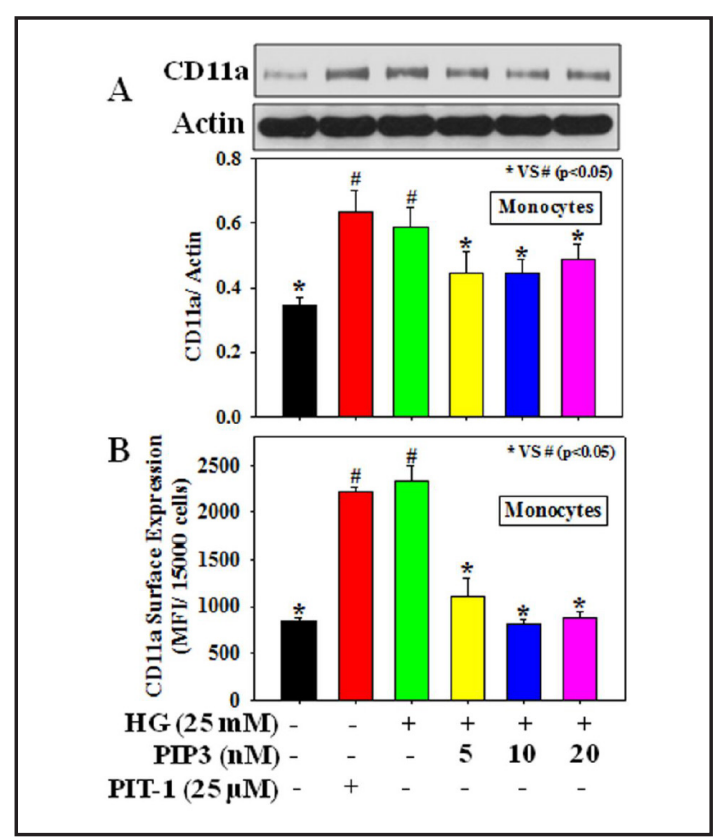

Fig. 3. Effect of PIP3 on CD11a (a subunit of LFA1) expression in THP-1 monocytes exposed to HG. A: CD11a total protein expression and B: CD11a surface expression. Cells were pretreated with PIP3 (5, 10, or $20 \mathrm{nM}$ ) for $4 \mathrm{~h}$ followed by HG (25 mM) exposure for the next $20 \mathrm{~h}$. Cells were also treated with the PIP3 inhibitor PIT-1 $(25 \mu \mathrm{M})$ for $20 \mathrm{~h}$. Values are expressed as mean \pm SE $(n=3)$. The difference between $*$ vs \# is considered significant at the $p<0.05$ level.

Fig. 4. Effect of PIP3 on the adhesion of monocytes to HUVEC treated with HG. Cells were pretreated with PIP3 $(5,10$, or $20 \mathrm{nM})$ for $4 \mathrm{~h}$ followed by HG (25 mM) exposure for the next $20 \mathrm{~h}$. Cells were also treated with the PIP3 inhibitor PIT-1 $(25 \mu \mathrm{M})$ for 20 $h$. Values are expressed as mean \pm SE $(n=3)$. The difference between ${ }^{*}$ vs \# is considered significant at the $p<0.05$ level.

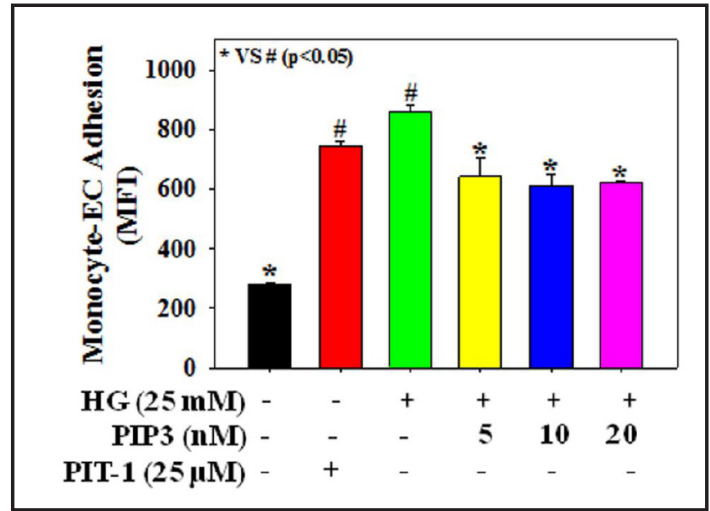

a significant increase in ICAM-1 total protein expression (2A) as well as its surface expression (2B) in HUVEC and CD11a total protein expression (3A) as well as its surface expression (3B) in THP-1 monocytes. Treatment with the PIP3 inhibitor PIT-1 also increased the expression of adhesion molecules in both HUVEC and monocytes. Exogenous PIP3 supplementation, however, downregulated the protein expression and surface expression of both ICAM-1 in HUVEC and CD11a in monocytes treated with HG.

The effect of PIP3 on the adhesion of monocytes to endothelial cells is shown in Figure 4. Cells treated with HG showed an increase in monocyte adhesion to endothelial cells. Treatment with PIT-1 also caused a similar increase in monocyte-EC adhesion. PIP3 supplementation, however, reduced the HG induced increase in monocyte-EC adhesion. This 
suggests that PIP3 plays a role in the regulation of monocyte adhesion to endothelial cells treated with HG.

\section{Discussion}

Phosphatidylinositol-3,4,5-triphosphate (PIP3) is a well-known lipid second messenger and has been implicated in the regulation of insulin signaling and glucose homeostasis. Tissue levels of PIP3 are low in type 1 and type 2 diabetic rats [21]. Recent studies have demonstrated the significant role played by endothelial dysfunction in the regulation of glucose homeostasis in diabetes [22, 23]. However, there is no report in the literature concerning whether PIP3 has a direct effect on endothelial dysfunction and vascular inflammation at the cellular level. This study demonstrates that treatment with HG or a PIP3 inhibitor, PIT-1, can cause a decrease in intracellular PIP3 levels and an increase in the expression of adhesion molecules as well as monocyte-EC adhesion. In addition, exogenous PIP3 supplementation prevented the expression of adhesion molecules and monocyte-EC adhesion in HG treated cells.

The intracellular PIP3 concentration is regulated by the PI3K/PTEN/SHIP equilibrium [24]. PIP3 binds to the pleckstrin homology domains (PH) of proteins, such as protein kinase B [25], 3-phosphoinositide-dependent kinases [26], protein kinase C [27], etc. and thereby regulates the activity and subcellular localization of a variety of signal transduction molecules. High glucose induced downregulation of PI3K/AKT signaling causes endothelial cell proliferative dysfunction [10]. High glucose concentration has also been reported to increase endothelial cell apoptosis [11], permeability [12], and monocyte-EC adhesion [13, 14]. This study for the first time demonstrates a reduction in intracellular PIP3 levels in HG treated monocytes as well as HUVEC. Treatment with a PIP3 inhibitor, PIT-1, also causes a similar decrease in cellular PIP3 levels in both monocytes and HUVEC. Exogenous PIP3 supplementation, however, restored the HG-induced loss in cellular PIP3 concentrations.

The vascular endothelium is a primary target for tissue damage by oxidants during inflammatory diseases including diabetes. Interactions of cell adhesion molecules (CAM) play a central role in mediating immune and inflammatory responses. Leukocyte functionassociated antigen-1 (LFA-1) is a member of the integrin-type family of CAM which is predominantly involved in leukocyte trafficking and extravasation [28]. It is composed of a common $\beta$-subunit (CD18) and a distinct $\alpha$-subunit (CD11a). LFA-1 is exclusively expressed on leukocytes and interacts with its primary ligand, ICAM-1, in endothelial cells to promote cell adhesion events required for normal and pathologic functions of the immune systems. Diabetic patients have higher blood levels of sICAM-1 or LFA-1 [29, 30]. Our study demonstrates that HG exposure causes an increase in both ICAM-1 total protein expression in HUVEC and CD11a total protein expression in monocytes. Surface analysis by FACS shows that HG treatment also upregulated the surface expression of ICAM-1 in HUVEC and CD11a in monocytes. Treatment with the PIP3 inhibitor PIT-1 causes a similar increase in total protein expression and surface expression of the adhesion molecules ICAM-1 in HUVEC and CD11a in monocytes. Activation of the vascular endothelium, increased adhesion of circulating monocytes to the injured endothelial layer, and their subsequent infiltration into the vessel wall and differentiation into macrophages are critical early events in the pathogenesis of atherosclerosis [31]. This study demonstrates that treatment with both HG and the PIP3 inhibitor PIT-1 increased the adhesion of monocytes to endothelial cells. Exogenous PIP3 supplementation causes a decrease in total protein expression as well as surface expression of adhesion molecules in both HUVEC and monocytes and thus reduces the adhesion of monocytes to endothelial cells treated with HG.

In conclusion, this study demonstrates that PIP3 plays an important role in the regulation of endothelial cell dysfunction and vascular inflammation. Diabetes is associated with impaired glucose metabolism and higher vascular inflammation. Using THP-1 monocytes and HUVEC, this study demonstrates for the first time that a decrease in PIP3 can cause 
activation of adhesion molecules and an increase in monocyte adhesion to endothelial cells treated with HG. On the other hand, exogenous supplementation with PIP3 prevented the activation of adhesion molecules and monocyte-EC adhesion during HG exposure. This suggests a causal role of PIP3 in the regulation of adhesion molecules and the adhesion of monocytes to HUVEC treated with HG.

\section{Acknowledgements}

The authors are supported by grants from NIDDK and the Office of Dietary Supplements of the National Institutes of Health R01 DK072433 and the Malcolm Feist Endowed Chair in Diabetes. This study is also funded by a fellowship from the Malcolm Feist Cardiovascular Research Endowment, LSU Health Sciences Center, Shreveport. The authors thank Ms Georgia Morgan for excellent editing of this manuscript.

\section{Disclosure Statement}

The authors declare that they have no conflict of interest.

\section{References}

1 Rains JL, Jain SK: Oxidative stress, insulin signaling, and diabetes. Free Radic Biol Med 2011;50:567-575.

- Hadi HA, Suwaidi JA: Endothelial dysfunction in diabetes mellitus. Vasc Health Risk Manag 2007;3:853-

876.

-3 Kearney MT, Duncan ER, Kahn M, Wheatcroft SB: Insulin resistance and endothelial cell dysfunction: studies in mammalian models. Exp Physiol 2008;93:158-163.

4 Rask-Madsen C, King GL: Mechanisms of Disease: endothelial dysfunction in insulin resistance and diabetes. Nat Clin Pract Endocrinol Metab 2007;3:46-56.

5 Rask-Madsen C, Li Q, Freund B, Feather D, Abramov R, Wu IH, Chen K, Yamamoto-Hiraoka J, Goldenbogen J, Sotiropoulos KB, Clermont A, Geraldes P, Dall'Osso C, Wagers AJ, Huang PL, Rekhter M, Scalia R, Kahn CR, King GL: Loss of insulin signaling in vascular endothelial cells accelerates atherosclerosis in apolipoprotein E null mice. Cell Metab 2010;11:379-389.

6 Pendaries C, Tronchere H, Plantavid M, Payrastre B: Phosphoinositide signaling disorders in human diseases. FEBS Lett 2003;546:25-31.

7 Okada T, Kawano Y, Sakakibara T, Hazeki O, Ui M: Essential role of phosphatidylinositol 3-kinase in insulin-induced glucose transport and antilipolysis in rat adipocytes. Studies with a selective inhibitor wortmannin. J Biol Chem 1994;269:3568-3573.

-8 Conricode KM: Involvement of phosphatidylinositol 3-kinase in stimulation of glucose transport by growth factors in 3T3-L1 adipocytes. Biochem Mol Biol Int 1995;36:835-843.

-9 Ghigo A, Perino A, Hirsch E: Phosphoinositides and cardiovascular diseases. Curr Top Microbiol Immunol 2012;362:43-60.

10 Varma S, Lal BK, Zheng R, Breslin JW, Saito S, Pappas PJ, Hobson RW, 2nd, Duran WN: Hyperglycemia alters PI3k and Akt signaling and leads to endothelial cell proliferative dysfunction. Am J Physiol Heart Circ Physiol 2005;289:H1744-1751.

-11 Liu B, Bhat M, Nagaraj RH: AlphaB-crystallin inhibits glucose-induced apoptosis in vascular endothelial cells. Biochem Biophys Res Commun 2004;321:254-258.

12 Dang L, Seale JP, Qu X: Reduction of high glucose and phorbol-myristate-acetate-induced endothelial cell permeability by protein kinase C inhibitors LY379196 and hypocrellin A. Biochem Pharmacol 2004;67:855864. 
13 Kim JA, Berliner JA, Natarajan RD, Nadler JL: Evidence that glucose increases monocyte binding to human aortic endothelial cells. Diabetes 1994;43:1103-1107.

14 Nandy D, Janardhanan R, Mukhopadhyay D, Basu A: Effect of hyperglycemia on human monocyte activation. J Investig Med 2011;59:661-667.

15 Franke TF, Kaplan DR, Cantley LC, Toker A: Direct regulation of the Akt proto-oncogene product by phosphatidylinositol-3,4-bisphosphate. Science 1997;275:665-668.

16 Jiang BH, Zheng JZ, Aoki M, Vogt PK: Phosphatidylinositol 3-kinase signaling mediates angiogenesis and expression of vascular endothelial growth factor in endothelial cells. Proc Natl Acad Sci U S A 2000;97:1749-1753.

17 McGinn S, Saad S, Poronnik P, Pollock CA: High glucose-mediated effects on endothelial cell proliferation occur via p38 MAP kinase. Am J Physiol Endocrinol Metab 2003;285:E708-717.

18 Candiloros H, Muller S, Zeghari N, Donner M, Drouin P, Ziegler O: Decreased erythrocyte membrane fluidity in poorly controlled IDDM. Influence of ketone bodies. Diabetes Care 1995;18:549-551.

19 Rains JL, Jain SK: Hyperketonemia increases monocyte adhesion to endothelial cells and is mediated by LFA-1 expression in monocytes and ICAM-1 expression in endothelial cells. Am J Physiol Endocrinol Metab 2011;301:E298-306.

20 Manna P, Jain SK: L-cysteine and hydrogen sulfide increase PIP3 and AMPK/PPARgamma expression and decrease ROS and vascular inflammation markers in high glucose treated human U937 monocytes. J Cell Biochem 2013;114:2334-2345.

21 Manna P, Jain SK: Decreased hepatic phosphatidylinositol-3,4,5-triphosphate (PIP3) levels and impaired glucose homeostasis in type 1 and type 2 diabetic rats. Cell Physiol Biochem 2012;30:1363-1370.

-22 Kim JA, Montagnani M, Koh KK, Quon MJ: Reciprocal relationships between insulin resistance and endothelial dysfunction: molecular and pathophysiological mechanisms. Circulation 2006;113:1888-1904.

-23 Pinkney JH, Stehouwer CD, Coppack SW, Yudkin JS: Endothelial dysfunction: cause of the insulin resistance syndrome. Diabetes 1997;46:S9-13.

24 Sasaki T, Sasaki J, Sakai T, Takasuga S, Suzuki A: The physiology of phosphoinositides. Biol Pharm Bull 2007;30:1599-1604.

-25 James SR, Downes CP, Gigg R, Grove SJ, Holmes AB, Alessi DR: Specific binding of the Akt-1 protein kinase to phosphatidylinositol 3,4,5-trisphosphate without subsequent activation. Biochem J 1996;315:709-713.

-26 Alessi DR, James SR, Downes CP, Holmes AB, Gaffney PR, Reese CB, Cohen P: Characterization of a 3-phosphoinositide-dependent protein kinase which phosphorylates and activates protein kinase Balpha. Curr Biol 1997;7:261-269.

27 Mellor H, Parker PJ: The extended protein kinase C superfamily. Biochem J 1998;332:281-292.

28 Simon SI, Green CE: Molecular mechanics and dynamics of leukocyte recruitment during inflammation. Annu Rev Biomed Eng 2005;7:151-185.

-29 Fogelstrand L, Hulthe J, Hulten LM, Wiklund O, Fagerberg B: Monocytic expression of CD14 and CD18, circulating adhesion molecules and inflammatory markers in women with diabetes mellitus and impaired glucose tolerance. Diabetologia 2004;47:1948-1952.

-30 Mysliwiec J, Kretowski A, Kinalski M, Kinalska I: CD11a expression and soluble ICAM-1 levels in peripheral blood in high-risk and overt type 1 diabetes subjects. Immunol Lett 1999;70:69-72.

31 Libby P: Inflammation in atherosclerosis. Nature 2002;420:868-874. 